\title{
Docking Studies of Derivates
} Phenylaminopyrimidines (PAP) as SARS-Cov-2 Main Protease Inhibitors ${ }^{\dagger}$

\author{
Abel Suárez-Castro *, Valeria Muñoz-Gutiérrez, Ma. Guadalupe Villa-Lopez, Claudia Contreras- \\ Celedón, Luis Chacón-García and Carlos J. Cortes-García * \\ Laboratorio de Diseño Molecular, Instituto de Investigaciones Químico Biológicas, Universidad Michoacana \\ de San Nicolás de Hidalgo, Ciudad Universitaria, C.P. 58033 Morelia, Mexico \\ + Presented at the 24th International Electronic Conference on Synthetic Organic Chemistry, 15 November- \\ 15 December 2020; Available online: https://ecsoc-24.sciforum.net/.
}

Received: date; Accepted: date; Published: date

\begin{abstract}
A set of 18 imine-phenylaminopyrimidines (imine-PAP) 5a-r against the main protease of SARS-CoV-2, is presented. In addition, these compounds have been previously reported by our group. The best receptor-ligand interactions were obtained from $10 \mathrm{i}, 10 \mathrm{~m}$ and $10 \mathrm{o}$ as shown by their predicted free Gibbs $-9.83,-9.71$ and $-9.02 \mathrm{kcal} / \mathrm{mol}$ respectively. This is in comparison with the cocrystalized ligand in the main protease $(-7.78 \mathrm{kcal} / \mathrm{mol}$,). These results provide solid foundation in order to test the imine-PAP compounds in in vitro studies in order to explore the possible inhibition of the main protease of SARS-CoV-2.
\end{abstract}

Keywords: phenylaminopyrimidines; molecular docking; main protease; SARS-CoV-2

\section{Introduction}

Coronavirus disease 2019 or COVID-19 is currently a pandemic disease caused by a new coronavirus strain known as SARS-CoV-2 that have infected and killed millions of people worldwide $[1,2]$. To date, there is no specific antiviral drug for the treatment of COVID-19 and the fastest solution has been drug repurposing. Only the remdesivir has been recently approved (authorized) by the FDA in the treatment for COVID-19, but only for treating certain patients [3-6]. Therefore, there are many efforts to find and develop a new specific antiviral drug. In this way, computer aided drug design (CADD) has played a special role in these efforts, being a powerful in silico tool to predict the possible interactions of small molecules in the active site of the principal target of SARS-CoV-2 namely Mpro protease [7-9]. Thus, molecular docking studies have been suitable for this objective as they confer, a reliable method to predict thousands and millions of small compounds that might inhibit this target SARS-Cov-2 [10].

On the other hand, phenylaminopyrimidines (PAP) are considered privileged nucleus because they have been utilized as a main component of many pharmaceutical drugs, such as imatinib $\mathbf{1}$, ceritinib 2, etravirine 3 and rilpivirine 4 (Figure 1) [11]. Notably, that last two drugs are used for the treatment of HIV-1 infections. Having in mind the above facts, we present molecular docking studies of a set of 18 imine-phenylaminopyrimidines (imine-PAP) 5a-r previously synthetized in our laboratory [12] against the main protease of SARS-CoV-2. 

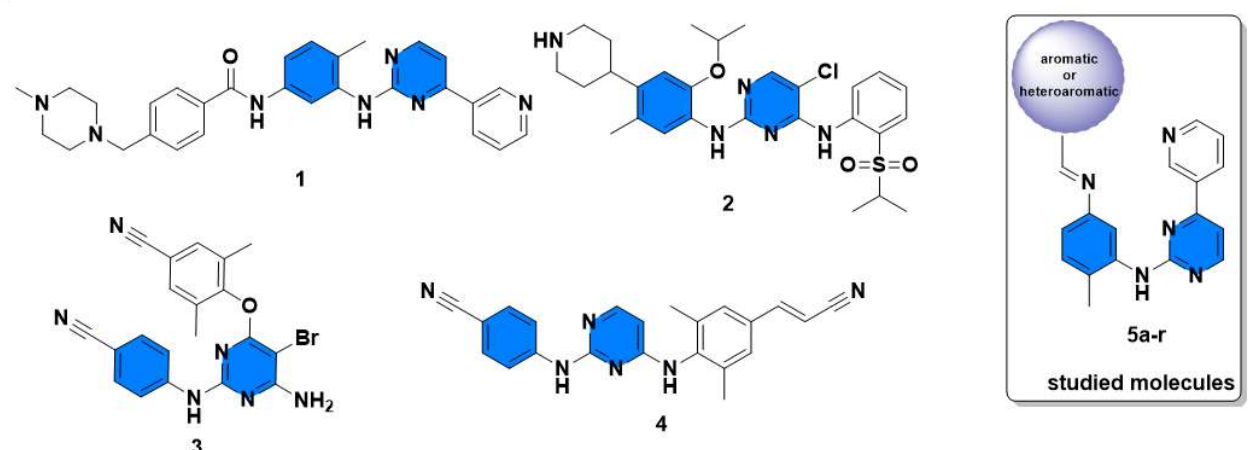

Figure 1. Studied molecules for molecular docking and drugs with PAP scaffold.

\section{Materials and Methods}

\subsection{Computational Details}

\subsubsection{Ligand Preparation}

The structures of compounds 5a-r (Figure 2) were modeled as 2D structures with the software ChemBio Drawltra 12.0 [13] and were converted into 3D structures in MDL format. Their protonated states were then computed using the online tool Chemicalize (www.chemicalize.org) [14]. The geometries of the compounds and co-crystalized ligand were calculated at the semiempirical AM1 level in the Gaussian 16 software package [15]. Finally, using Autodock Tools [16], the ligands were prepared by adding polar hydrogens and Gasteiger charges and rotatable (i.e., single) bonds were assigned by default, and a pdbqt file was generated.

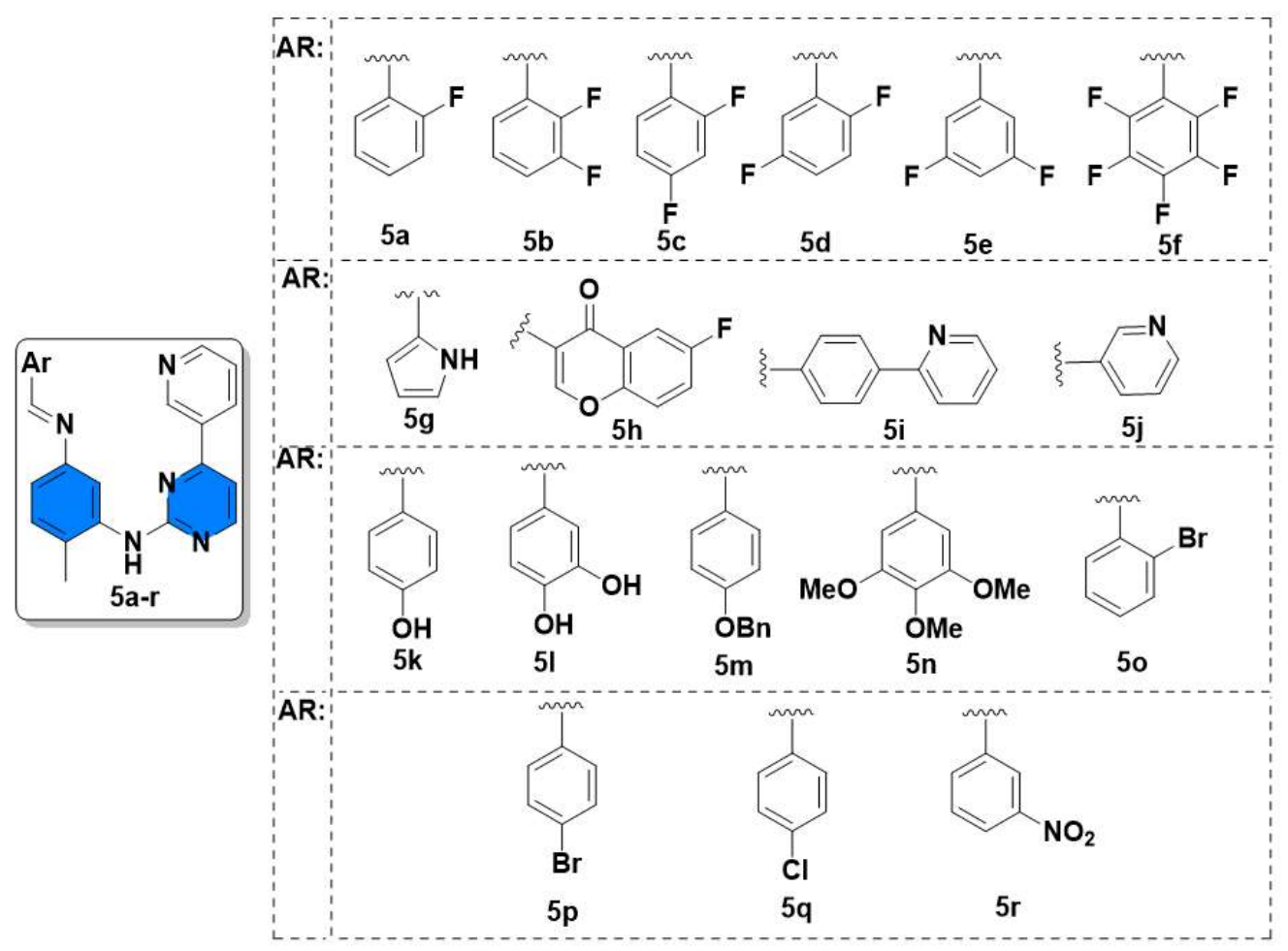

Figure 2. Phenylaminopyrimidines 5a-r evaluated as SARS-Cov-2 main protease inhibitors. 


\subsubsection{Receptor Preparation}

The X-ray coordinates of the Mpro protease receptor of SARS-CoV-2 were retrieved from the Protein Data Bank (PDB code:6LU7) [17]. Molecular water was removed from the crystallographic structure and the final preparation and minimization of the receptor structure was carried out using the Dock Prep module of Chimera software [18] using the AMBER-ff14SB force field. Lastly, Kollman charges were added using Autodock Tools and a pdbqt file was generated.

\subsubsection{Docking Calculations}

Rigid receptor molecular docking was carried out in Autodock4 using the Lamarckian genetic algorithm [19]. We used grid maps with $70 \times 70 \times 70$ points in the active site of the receptor with the coordinates $\mathrm{x}=-12.202, \mathrm{y}=11.499, \mathrm{z}=69.669$, and a grid-point spacing of $0.375 \AA$. AD4.dat parameters were applied to all of the ligands. The parameters used were 10 runs, a population size of 100 , and a run-termination criterion of a maximum of 27,000 generations or a maximum of 10,000,000 energy evaluations. The visualization and analysis of the nonbonded interactions as hydrogen bonds of the best poses were carried out using Discovery Studio Visualizer software [20].

\section{Results and Discussion}

To explore the possible modes of interaction of compounds $\mathbf{5 a - r}$ against Mpro protease receptor of SARS-CoV-2, molecular docking studies were carried out according with the specifications redacted. The predicted free energy and affinity constant values are listed in Table 1, including the predicted free energy for the co-crystalized ligand (N3) in the PDB code 6LU7 [21].

Table 1. Free energy $(\Delta \mathrm{G})$ and affinity constant (ki and $p \mathrm{ki}$ ) values predicted from the results of molecular dockings of compounds $5 \mathbf{a}-\mathbf{r}$ with Mpro protease receptor of SARS-CoV-2.

\begin{tabular}{cccc}
\hline Compound & $\Delta \mathbf{G} \mathbf{( k c a l} / \mathbf{m o l})$ & $\mathbf{k i} \mathbf{( n M})$ & $p \mathbf{k i}$ \\
$\mathbf{5 a}$ & -8.17 & 1,030 & 5.99 \\
$\mathbf{5 b}$ & -8.21 & 961.56 & 6.02 \\
$\mathbf{5 c}$ & -8.34 & 772.92 & 6.11 \\
$\mathbf{5 d}$ & -8.67 & 443.92 & 6.35 \\
$\mathbf{5 e}$ & -8.28 & 859.13 & 6.07 \\
$\mathbf{5 f}$ & -7.87 & 1,710 & 5.77 \\
$\mathbf{5 g}$ & -8.19 & 999.33 & 6.00 \\
$\mathbf{5 h}$ & -8.93 & 286.58 & 6.54 \\
$\mathbf{5 i}$ & -9.83 & 62.73 & 7.20 \\
$\mathbf{5 j}$ & -8.90 & 297.89 & 6.53 \\
$\mathbf{5 k}$ & -7.97 & 1,440 & 5.84 \\
$\mathbf{5 1}$ & -7.97 & 1,440 & 5.84 \\
$\mathbf{5 m}$ & -9.71 & 75.81 & 7.12 \\
$\mathbf{5 n}$ & -8.50 & 587.57 & 6.23 \\
$\mathbf{5 0}$ & -9.02 & 243.2 & 6.61 \\
$\mathbf{5 p}$ & -8.47 & 621.18 & 6.21 \\
$\mathbf{5 q}$ & -8.47 & 614.61 & 6.21 \\
$\mathbf{5 r}$ & -8.29 & 833.27 & 6.08 \\
$\mathbf{N} 3$ & -7.78 & 1,700 & 2.77 \\
\hline
\end{tabular}


Compounds 5a-r showed good predicted free energy compared with the N3 ligand. It is important to note that the N3 is a peptide (Figure 3.) and it has been tested against other SARS-CoV and other MERS-CoV viruses [22] with good outcomes of inhibition for Mpro. The key reported amino acids in the active site are His41 and Cys145, which play a role as a catalytic dyad, and where the main efforts are focused to find Mpro inhibitors.

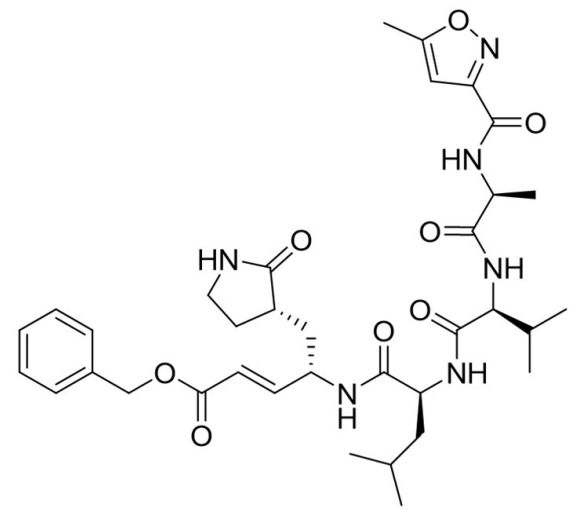

Figure 3. Co-crystalized N3 ligand in 6LU7.

According to our docking results, five of PAP compounds showed the best predicted free energy: $\mathbf{5 h}, \mathbf{5 i}, \mathbf{5 j}, \mathbf{5 m}$ and $\mathbf{5 o}$ (Figure 4 ) and their substituents at the aromatic or heteroaromatic scaffolds have $\pi$-electrons that contribute to form the main interactions between ligands and the active site of the receptor

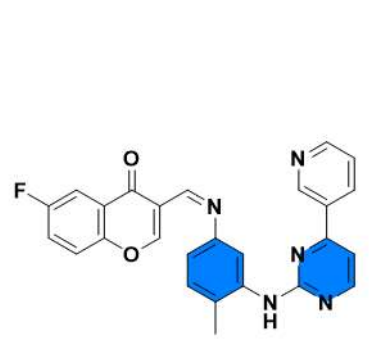

$5 \mathrm{~h}$

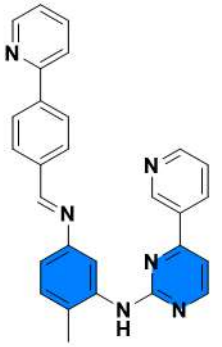

$5 \mathbf{i}$

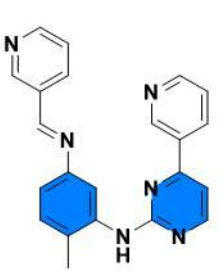

$5 \mathbf{j}$

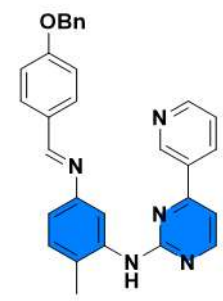

$5 \mathrm{~m}$

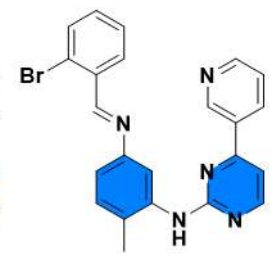

50

Figure 4. Phenylaminopyrimidines with the best predicted free energies and ki values.

The principal interactions of the best predicted free energy compounds are depicted in Table 2. The PAP moiety showed a hydrogen bond almost in all the tested compounds with Gly143, His164, and Glu166, being the most important predicted interaction in our in silico results. On the other hand, the same PAP moiety showed, in some cases, a $\pi$-anion or $\pi$-sigma interaction with Glu166 becoming the second most important predicted interaction. Compounds $5 \mathbf{i}$ and $5 \mathrm{~m}$ showed the best free energy predicted ( -9.83 and $-9.71 \mathrm{kcal} / \mathrm{mol}$ respectively) and possess an aromatic 4-phenyl pyridine and a O-benzyl moiety, respectively. These aromatic scaffolds contain $\pi$ electrons that could interact with other $\pi$-systems or with electron rich atoms such as sulphur in cysteine. 
Table 2. Results of docking studies of 5a-r Phenylaminopyrimidines.

\begin{tabular}{|c|c|c|}
\hline Compound & Residues of Interaction & Type of Interaction \\
\hline \multirow{3}{*}{$5 h$} & Gly143 & Hydrogen bond \\
\hline & His41 & $\pi-\pi$ \\
\hline & Glu166 & $\pi$-anion \\
\hline \multirow{4}{*}{$5 i$} & Gly143 & Hydrogen bond \\
\hline & His41 & $\pi-\pi$ \\
\hline & Met49 & $\pi$-sulfur \\
\hline & Glu166 & $\pi$-sigma \\
\hline \multirow{4}{*}{$5 \mathbf{j}$} & His41 & $\pi-\pi$ \\
\hline & Cys145 & $\pi$-sulfur \\
\hline & His164 & Hydrogen bond \\
\hline & Met165 & $\pi$-sulfur \\
\hline \multirow{3}{*}{$5 \mathrm{~m}$} & Glu166 & Hydrogen bond \\
\hline & Cys145 & $\pi$-sulfur \\
\hline & Met165 & $\pi$-sulfur \\
\hline \multirow{3}{*}{50} & Glu166 & Hydrogen bond \\
\hline & His163 & Hydrogen bond \\
\hline & Met165 & $\pi$-sulfur \\
\hline
\end{tabular}

It is important to highlight that $\mathbf{5 h}, \mathbf{5 i}$ and $\mathbf{5} \mathbf{j}$ showed $\pi-\pi$ interactions with His41 (Figure $5 \mathrm{~A}-$ C), a key amino acid in the active site, contributing with approximately $2-3 \mathrm{kcal} / \mathrm{mol}$ to the ligandreceptor interaction.

Conversely, $\mathbf{5 j}$ and $\mathbf{5 m}$ showed a $\pi$-sulfur interaction with the Cys145 residue. This kind of interactions are not common in the ligand-receptor poses and show that they could contribution to the possible inhibition of Mpro.

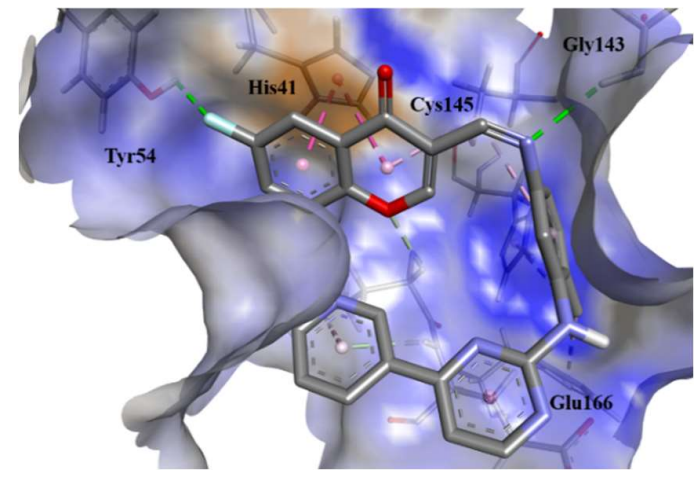

(A)

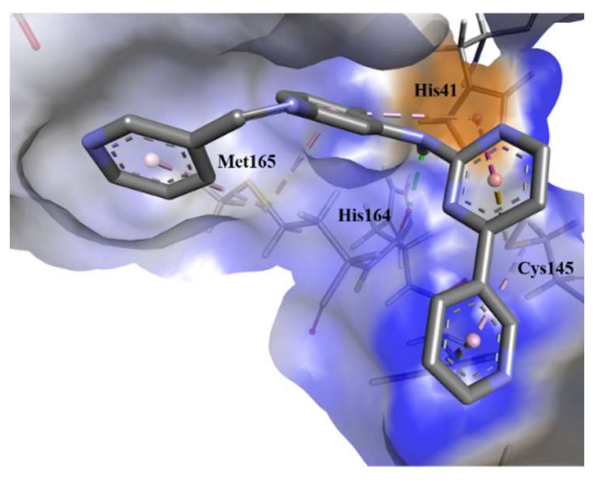

(C)

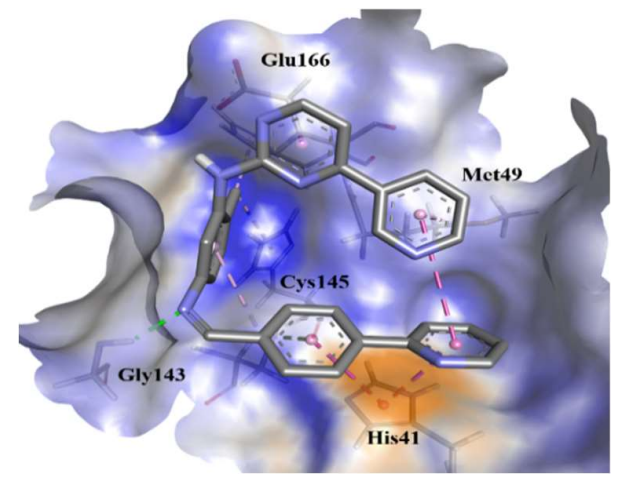

(B)

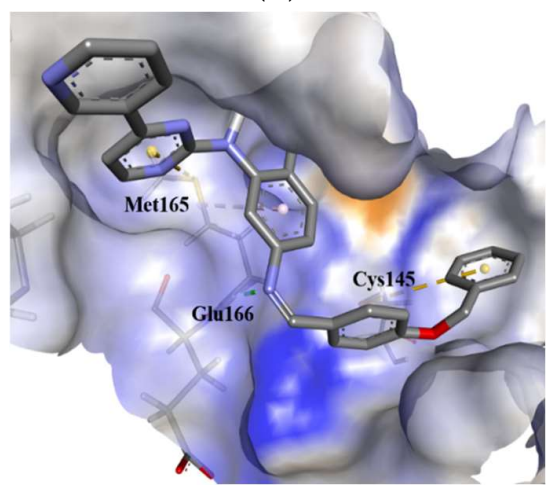

(D) 


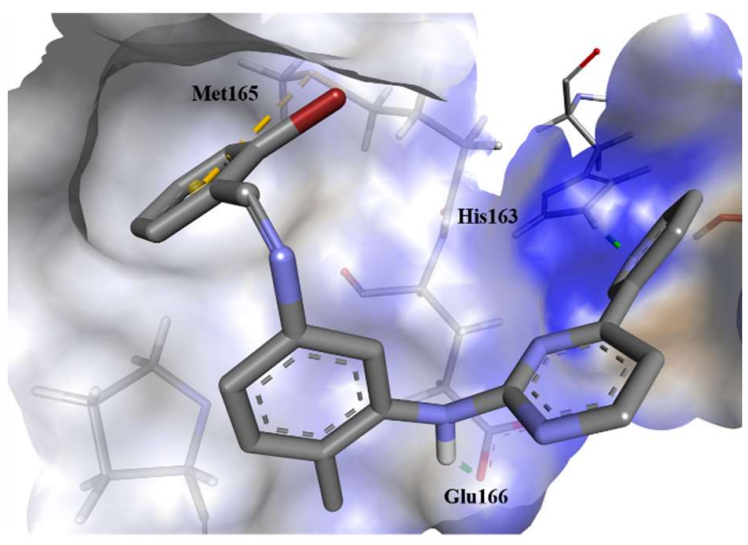

(E)

Figure 5. Poses of the best predicted PAP ligands into the active site of Mpro protease of SARS-CoV-2.

(A): $5 \mathbf{h} ;(B): 5 i ;(C): 5 j,(D): 5 m ;(E): 5 o$.

\section{Conclusions}

The importance of the design and development of inhibitors against SARS-CoV-2 have become a priority in the pharmaceutical sphere. Many efforts are in progress and in many cases computer aided drug discovery is a tool to start with the identification of possible inhibitors in any step of SARS-CoV-2 replication cycle. Here, we tested in silico 18 PAP compounds synthetized in our laboratory showing the predicted free energy and probable ligand-receptor interactions using molecular docking approaches. The major interactions were presented by the PAP moiety and the aromatic or heteroaromatic scaffold stablishing $\pi-\pi$ or $\pi$-sulfur interactions. These results pave the way to carry in vitro assays and better determine their activity as probable Mpro inhibitors.

Author Contributions: All authors contribute equally to the work.

Acknowledgments: Carlos C.-G. is grateful for financial support from CIC-UMSNH (14646).

Conflicts of Interest: The authors declare no conflicts of interest or state

\section{References}

1. Khan, Z.; Karataş, Y.; Rahman, H. Anti COVID-19 Drugs: Need for More Clinical Evidence and Global Action. Adv. Ther. 2020, 37, 2575-2579, doi:10.1007/s12325-020-01351-9.

2. Harapan, H.; Itoh, N.; Yufika, A.; Winardi, W.; Keam, S.; Te, H.; Megawati, D.; Hayati, Z.; Wagner, A.L.; Mudatsir, M. Coronavirus disease 2019 (COVID-19): A literature review. J. Infect. Public Health 2020, 13, 667-673, doi:10.1016/j.jiph.2020.03.019.

3. ElFiky, A.A. Ribavirin, Remdesivir, Sofosbuvir, Galidesivir, and Tenofovir against SARS-CoV-2 RNA dependent RNA polymerase (RdRp): A molecular docking study. Life Sci. 2020, 253, 117592, doi:10.1016/j.lfs.2020.117592.

4. ElFiky, A.A. Anti-HCV, nucleotide inhibitors, repurposing against COVID-19. Life Sci. 2020, 248, 117477, doi:10.1016/j.lfs.2020.117477.

5. Li, G.; De Clercq, E. Therapeutic options for the 2019 novel coronavirus (2019-nCoV). Nat. Rev. Drug Discov. 2020, 19, 149-150, doi:10.1038/d41573-020-00016-0.

6. Coronavirus Disease 2019 (COVID-19). Available online: https://www.fda.gov/emergency-preparednessand-response/counterterrorism-and-emerging-threats/coronavirus-disease-2019-covid-19 (accessed on 3 November 2020).

7. Onawole, A.T.; Sulaiman, K.O.; Kolapo, T.U.; Akinde, F.O.; Adegoke, R.O. COVID-19: CADD to the rescue. Virus Res. 2020, 285, 198022, doi:10.1016/j.virusres.2020.198022.

8. Tabeshpour, J.; Sahebkar, A.; Zirak, M.R.; Zeinali, M.; Hashemzaei, M.; Rakhshani, S.; Rakhshani, S. Computer-aided Drug Design and Drug Pharmacokinetic Prediction: A Mini-review. Curr. Pharm. Des. 2018, 24, 3014-3019, doi:10.2174/1381612824666180903123423. 
9. Ferreira, L.G.; Dos Santos, R.N.; Oliva, G.; Andricopulo, A.D. Molecular Docking and Structure-Based Drug Design Strategies. Molecules 2015, 20, 13384-13421, doi:10.3390/molecules200713384.

10. Cortés-García, C.J.; Chacón-García, L.; Mejía-Benavides, J.E.; Díaz-Cervantes, E. Tackling the SARS-CoV-2 main protease using hybrid derivatives of 1,5-disubstituted tetrazole-1,2,3-triazoles: An in silico assay. PeerJ Phys. Chem. 2020, 2, e10, doi:10.7717/peerj-pchem.10.

11. Wishart, D.S.; Feunang, Y.D.; Guo, A.C.; Lo, E.J.; Marcu, A.; Grant, J.R.; Sajed, T.; Johnson, D.; Li, C.; Sayeeda, Z.; et al. DrugBank 5.0: A major update to the DrugBank database for 2018. Nucleic Acids Res. 2018, 46, D1074-D1082, doi:10.1093/nar/gkx1037.

12. Cortés-García, C.J.; Chacón-García, L.; Gámez-Montaño, R. In Proceedings of the 21th Int. Electron. Conf. Synth. Org. Chem., MDPI AG, 2017; In Sciforum Electronic Conference Series; Volume 21, p 1.

13. Cousins, K.R. Computer Review of ChemDraw Ultra 12.0. J. Am. Chem. Soc. 2011, 133, 8388, doi:0.1021/ja204075s.

14. Swain, M.C. chemicalize.orgchemicalize.orgby ChemAxon Ltd. J. Chem. Inf. Model. 2012, 52, 613-615, doi:10.1021/ci300046g.

15. Gaussian 16; Revision C.01; Frisch, M.J.; Trucks, G.W.; Schlegel, H.B.; Scuseria, G.E.; Robb, M.A.; Cheeseman, J.R.; Scalmani, G.; Barone, V.; et al. Gaussian, Inc.: Wallingford, CT, USA, 2016.

16. Sanner, M.F. A Programming Language for Software Integration and Development. J. Mol. Graph. Model. 1999, 17, 55-84, doi:10.1016/s1093-3263(99)99999-0.

17. Rose, P.W.; Prlić, A.; Bi, C.; Bluhm, W.F.; Christie, C.H.; Dutta, S.; Green, R.K.; Goodsell, D.S.; Westbrook, J.D.; Woo, J.; et al. The RCSB Protein Data Bank: Views of structural biology for basic and applied research and education. Nucleic Acids Res. 2015, 43, D345-D356, doi:10.1093/nar/gku1214.

18. Pettersen, E.F.; Goddard, T.D.; Huang, C.C.; Couch, G.S.; Greenblatt, D.M.; Meng, E.C.; Ferrin, T.E. UCSF Chimera-A visualization system for exploratory research and analysis. J. Comput. Chem. 2004, 25, 16051612, doi:10.1002/jcc.20084.

19. Morris, G.M.; Goodsell, D.S.; Halliday, R.S.; Huey, R.; Hart, W.E.; Belew, R.K.; Olson, A.J. Automated docking using a Lamarckian genetic algorithm and an empirical binding free energy function. J. Comp. Chem. 1998, 19, 1639-1662, doi.:10.1002/(SICI)1096-987X(19981115)19:143.0.CO;2-B.

20. BIOVIA. Dassault Systèmes, Discovery Studio 2019; Dassault Systèmes: San Diego, CA, USA, 2019.

21. Jin, Z.; Du, X.; Xu, Y.; Deng, Y.; Liu, M.; Zhao, Y.; Zhang, B.; Li, X.; Zhang, L.; Peng, C.; et al. Structure of Mpro from SARS-CoV-2 and discovery of its inhibitors. Nat. Cell Biol. 2020, 582, 289-293, doi:10.1038/s41586-020-2223-y.

22. Yang, H.; Xie, W.; Xue, X.; Yang, K.; Ma, J.; Liang, W.; Zhao, Q.; Zhou, Z.; Pei, D.; Ziebuhr, J.; et al. Design of Wide-Spectrum Inhibitors Targeting Coronavirus Main Proteases. PLoS Biol. 2005, 3, e324, doi:10.1371/journal.pbio.0030324.

Publisher's Note: MDPI stays neutral with regard to jurisdictional claims in published maps and institutional affiliations. 